\title{
THE STRUCTURE AND EVOLUTION OF X-RAY CLUSTERS OF GALAXIES
}

\author{
C. Jones \\ Harvard/Smithsonian Astrophysical Observatory
}

Rich clusters are the largest, well-studied aggregates of matter in the universe. It is a basic observational fact that the distribution of galaxies on the sky shows a high degree of clumpiness (Fig. 1). A cluster is essentially an enhancement in the density of galaxies projected on the sky. Abell (1958), who has done much of the basic work of systemizing the concept of cluster, includes in his catalog clusters that have at least 50 galaxies in a particular magnitude range and $3 \mathrm{Mpc}$ radius of the cluster center. For richer clusters, there may be more than several hundred galaxies within this radius. Several schemes have been suggested to classify clusters with different morphology. The cluster in Figure 1 is the Coma cluster $(138 \mathrm{Mpc})$ and is a so-called "binary" system dominated by a pair of giant galaxies.

One of the main types of clusters that figures prominently in the realm of $\mathrm{X}$-ray clusters is called a $\mathrm{cD}$ cluster. These clusters are dominated by a single giant of $\mathrm{cD}$ type galaxy. $\mathrm{cD}^{\prime} \mathrm{s}$ are the largest galaxies known and have the core of a giant elliptical galaxy and an extended envelope of low surface brightness. Ostriker and his coworkers have suggested that these galaxies are formed by a process in which massive galaxies in a dense cluster core lose energy by dynamical friction as they move towards equipartition with less massive galaxies; consequently, they spiral in towards the center of the cluster's potential well. There, the cD's grow by cannibalizing other galaxies whose envelopes approach nearby. This theory predicts that $\mathrm{cD}$ galaxies will be found at or near the cluster center. The Einstein X-ray observations, can be used to determine the cluster center and can even improve on galaxy counts as a means of determining the center. We therefore can compare the X-ray center and the position of the $\mathrm{cD}$ galaxy.

Figure 2 gives an example of a cluster that has quite a different central structure from both Coma and $\mathrm{cD}$ clusters. This is the Perseus cluster (at a distance of $110 \mathrm{Mpc}$ ), in which several bright galaxies are spread out along a line.

Figure 3 shows a result for the Perseus cluster prodiced by Forman and colleagues from the first X-ray satellite, Uhuru. Indicated are the contours of an extended radio source associated with the cluster, tailed radio sources around galaxies, as well as the error ellipse and extent for the $X$-ray source. Observations of this type showed for the first time that $X$-ray emission from clusters originates in a diffuse region (radius $\sim 15$ arc min) rather than a point-like source. This 
ability to distinguish between a point and an extended source was about the limit of the Uhuru, SAS, and HEAO-1 satellites, and then that analysis was only possible for very bright, nearby clusters. However, now the era of imaging allows one to study the detailed structure of clusters.

Let's begin with Einstein imaging observations of the nearby Virgo cluster which is being studied by Forman and colleagues. These observations were made with the Imaging Proportional Counter (IPC) at the focus of the Einstein $\mathrm{X}$-ray Observatory. The observations cover the energy range from approximately 0.5 to $3.0 \mathrm{keV}$, and have a spatial resolution of about 2 arc min. Figure 4 shows one of the fields in the Virgo cluster. There are five sources in this one square degree field. All but the bottom one are associated with galaxies. From this "figure it is apparent that one source is extended. Also there is more emission on the left edge than on the bottom edge. This diffuse emission is associated with the giant elliptical galaxy M87. Fabricant and co-workers are analyzing the imaging observations of M87 and can trace this extended emission out to more than a degree from the galaxy.

Figure 5 shows the contour plot of this field superposed on a Kitt Peak 4-meter photograph. The X-ray emission is clearly associated with the galaxies. Their luminosities range from a few times $10^{40} \mathrm{ergs} / \mathrm{sec}$ to a few times $10^{41} \mathrm{ergs} / \mathrm{sec}$ for $\mathrm{M} 86$. The total emission from our galaxy or Andromeda is a few times $10^{39} \mathrm{ergs} / \mathrm{sec}$. The emission from M86 appears diffuse and extended by as much as 10 arc min. Its isothermal core radius corresponds to $20 \mathrm{kpc}$.

This is an interesting group of galaxies. M84 contains a 3C radio source. The galaxy NGC 4388 is an ARP object and appears tidally disrupted by its companion. The other spiral (NGC 4438) was observed by $W$. Liller with the $\mathrm{Z}$-machine at Mt. Hopkins and found to be an emission line galaxy. Based on optical and radio information, one might have concluded that M86 was the least unusual. However, these X-ray observations show that M86 is also worthy of study.

The discovery of diffuse $\mathrm{X}$-ray emission from clusters and the realization, primarily from the discovery of iron emission by Midell and Serlemitsos and their colleagues, that most of the observed emission was due to thermal bremsstrahlung, brought about the question of the origin of the hot gas. Two basic types of models involved either the infall of primordial gas into the cluster from the intergalactic medium (Gunn and Gott 1972) or the injection (or stripping) of evolved gas in galaxies to form the intracluster medium (Yahil and Ostriker 1973, and Cowie and Binney 1977). The iron emission lines indicated that roughly solar abundances of iron were present and provided support for the models in which the $\mathrm{X}$-ray cluster emission is produced by gas which has been processed in stars and then subsequently lost to the intracluster region. 
Earlier observations have presented a picture of the Virgo cluster $\mathrm{X}$-ray emission consisting of two components - a cool $2 \mathrm{keV}$ gas contained by the gravitational potential of M87, and a hotter $(\sim 10 \mathrm{keV})$ gas of lower density contained by the gravitational potential of the entire cluster. The hot material must have a low density $\left(\sim 10^{-4} \mathrm{~cm}^{-3}\right)$ to account for its low surface brightness. This hot gas could be either primordial, or a result of injection since we have no detailed positional and spectral information which would determine its abundances and thereby its origin.

The Einstein observations have added to this picture and show that cool gas surrounds several of the Virgo galaxies. Embedded in : such a system, the gas surrounding M86 originated within M86iitself, rather than from infall of intracluster gas, since the hot intracluster gas could not cool sufficiently rapidly. Second, our preliminary spectral analysis of the emission implies that the gas around M86 is similar to that associated with M87 and has a cool temperature of $\sim 1 \mathrm{keV}$, and that it contains line emission and is therefore evolved. Although most of these galaxies have unusual optical or radio properties which may account for their X-ray emission, another possibility is that all these galaxies like M87 and M86 are gravitationally binding some of the cool gas.

The Virgo cluster phenomenon of gas associated with galaxies is not unique. We have studied several nearby rich clusters of galaxies with the Einstein Observatory IPC. These observations have shown that clusters display a variety of structures in their X-ray surface brightness distributions. We will discuss our observations in terms of the theoretical models for dynamic evolution in clusters. We have selected A85 and A1367 to discuss since they are representative of the types of clusters which have been observed.

Figure 6 shows the IPC observation of A1367. The cluster fills much of the IPC image. We have projected the flux onto each axis to illustrate the broad, extended cluster profile. From this picture it is also apparent that the cluster emission is elongated. At one end of the cluster is the radio galaxy $3 \mathrm{C} 264$ whose $\mathrm{X}$-ray emission is seen above that of the cluster, although its luminosity is only a few percent that of the cluster's.

Figure 7 shows a contour plot of the X-ray emission in A1367 superposed on an enlargement from the Palomar Sky Survey. The bright source at the bottom is $3 \mathrm{C} 264$ whose 0.25 to $3.0 \mathrm{keV}$ luminosity is a few (2) times $1042 \mathrm{ergs} / \mathrm{sec}$. The strong radio emission in this galaxy suggests unusual activity and, therefore, a higher X-ray luminosity than for other cluster members. Several other galaxies can be associated with regions of enhanced $X$-ray emission. The two galaxies, NGC 3842 and NGC 3841 , have $X$-ray luminosities of several (3.5 and 5.5$)$ times $10^{42}$ ergs/sec. These luminosities are about 100 times greater than the X-ray luminosity of our galaxy or Andromeda, but comparable to the luminosity of M86 in the Virgo cluster. 
This contour plot shows that the X-ray emission is highly clumped. Although some of the $X$-ray fluctuations correspond to bright galaxies, others do not. Given a relation between the mass of a galaxy and the associated $\mathrm{X}$-ray emission, the enhancements which do not correlate with bright galaxy may indicate the presence of previously undetected mass in the cluster.

Figure 8 shows the IPC observation of A 85 for which we have again shown the image projected onto each axis. Unlike the broad surface brightness profile for A1367, for A85 the emission is sharply peaked.

Figure 9 shows the contour plot for A85 on the Palomar print. The peaked of the emission coincides with the giant $c D$ galaxy. The $\mathrm{X}$-ray emission of this cluster is concentrated toward the cluster center and does not show the clumping that was so apparent for A1367.

Of the clusters which we have studied with the Einstein observatory, several have shown broad, highly clumped X-ray emission, while for others the emission is smooth, and centrally peaked.

Although time does not permit a detailed discussion of each cluster, we can use the broad versus peaked features of the surface brightness distribution to characterize the cluster. Figure 10 shows the radial distribution of the $\mathrm{X}$-rays for several clusters. The angular dimensions have been converted to linear size with the axis ending at $1 \mathrm{Mpc}$. We have also normalized the clusters to the same peak central intensities to illustrate their different extents. For these clusters, which all contain $\mathrm{cD}$ galaxies, the emission is centrally peaked while for the others, the emission is broader. Contour plots of these extended clusters also show that the emission often is clumped although for a few clusters with extended profiles, the emission is smooth.

These observations can be compared with the models for dynamic evolution in clusters. Through numerical simulations, Peebles, Aarseth and White have shown that a cluster which begins as a large cloud of galaxies will collapse and often subcluster and finally reach equilibrium with an extended halo around a dense core. The broad, highly clumped clusters could be interpreted as clusters in their early evolutionary stages in which the gas escaping the galaxies is bound more by the gravitational potential of the individual galaxies than by the relatively weak potential field of the cluster. We can use the cluster velocity dispersion as a measure of the cluster potential. Clusters with low velocity dispersion would have weak cluster potentials and the potentials of individual galaxies would cause substantial deviation from a smooth surface brightness distribution. The Virgo cluster and the bright galaxy group in A1367 have low velocity dispersions - less than half the value measured for Perseus (Noonal 1973, Tifft and Tarenghi 1973). 
Both clusters show substantial clumping of the X-ray luminosity around galaxies. Clusters in this early stage would be expected to have a lower density of hot intracluster gas and, therefore, a hîgher fraction of spirals than in more evolved clusters in which the ram pressure of the gas would strip the galaxies of their interstellar matter (Gunn and Gott 1972, Gisler 1976) thereby transforming spirals into SO's. Bahcall (1978) has determined the percentage of spirals in A1367 to be 40 percent which is considerably higher than the $\sim 10$ percent spirals she found in Coma and Perseus.

During the second phase of cluster evolution, a high density core or subcluster is formed, thereby enhancing the chances for building a cD galaxy at the cluster center. The clusters in our rather limited sample which show strongly peaked $\mathrm{X}$-ray radiation are all Bautz-Morgan type I clusters. These $\mathrm{cD}$ clusters have greater central surface brightnesses than do the less evolved clusters. This increased surface brightness is probably due to the increased gas density at the cluster center as the gas becomes bound by the cluster potential.

In the final stage of cluster evolution, the cluster has reached equilibrium. For this phase, we would expect the $\mathrm{X}$-ray emission to follow the cluster potential as traced by the galaxies. Since the gas distribution is dominated by the cluster potential, it should be smooth. Also, the percentage of spiral galaxies should remain small. Coma and the $\mathrm{cD}$ clusters are possible examples of clusters in this final stage. We have found that the gas distribution in the $\mathrm{cD}$ clusters is quite smooth. The surface brightness distribution of Coma and other evolved clusters such as A2256 is also smooth, although not as centrally peaked as the $\mathrm{eD}$ clusters.

In summary, we have found from the first observations of the structure of clusters with the Einstein Observatory that the nature of the $\mathrm{X}$-ray emission is complex and varies from broad and highly clumped, to smooth and centrally peaked. The clusters whose emission is clumped tend to be rich in spirals and to have $\mathrm{X}$-ray temperatures in the few kilovolt range and low velocity dispersions. The smooth, centrally peaked clusters are spiral poor, and have higher temperatures and larger velocity dispersions. For many of the clusters, the emission is irregular and cannot be described by the simple, spherically symmetric models for a hot isothermal or adiabatic cluster gas. For these clusters, the low density, intracluster gas is ..strongly influenced by the potential of individual bright galaxies.

The observed X-ray structure combined with optical properties such as the spiral fraction and central density complement the theoretical model for dynamic evolution in clusters. 
Many of the X-ray properties of clusters, including their structure and luminosity appear to be strongly influenced by their dynamical evolution. However, since clusters do not all evolve at the same rate due to differences in cluster properties such as richness, we are able to observe clusters at the same epoch but in a variety of evolutionary phases.

We were surprised by the complexity and variety in the surface brightness of nearby clusters. This great variety complicated the search for evolutionary changes with distance. Figure 11 shows the IPC image of 3C 295 which is one of the distant clusters studied by Pat Henry and co-workers. This cluster has a redshift of 0.46 and is one of the several distant clusters observed with Einstein. Optical observations of this cluster showed that although there was a dominant galaxy, and therefore the cluster should be evolved, about half the galaxies were spirals which is larger than would be expected in a centrally condensed cluster. One possible explanation for the high spiral percentage had been that there was no intergalactic medium to strip the spirals of their gas and thus, convert them to SO's. However, the observation of extended $\mathrm{X}$-ray emission from this cluster rules out that possibility. Another possibility which is consistent with the observation is that in this distant cluster with its young galaxies, the rate of gas injection from stars is high. This high rate could slow the stripping process and cause spirals to remain spirals longer.

Thus, to summarize the Einstein observations of clusters it appears, somewhat ironically, that we are learning about the evolution of young galaxies in distant clusters and about the evolution of clusters from our observations of nearby clusters. 


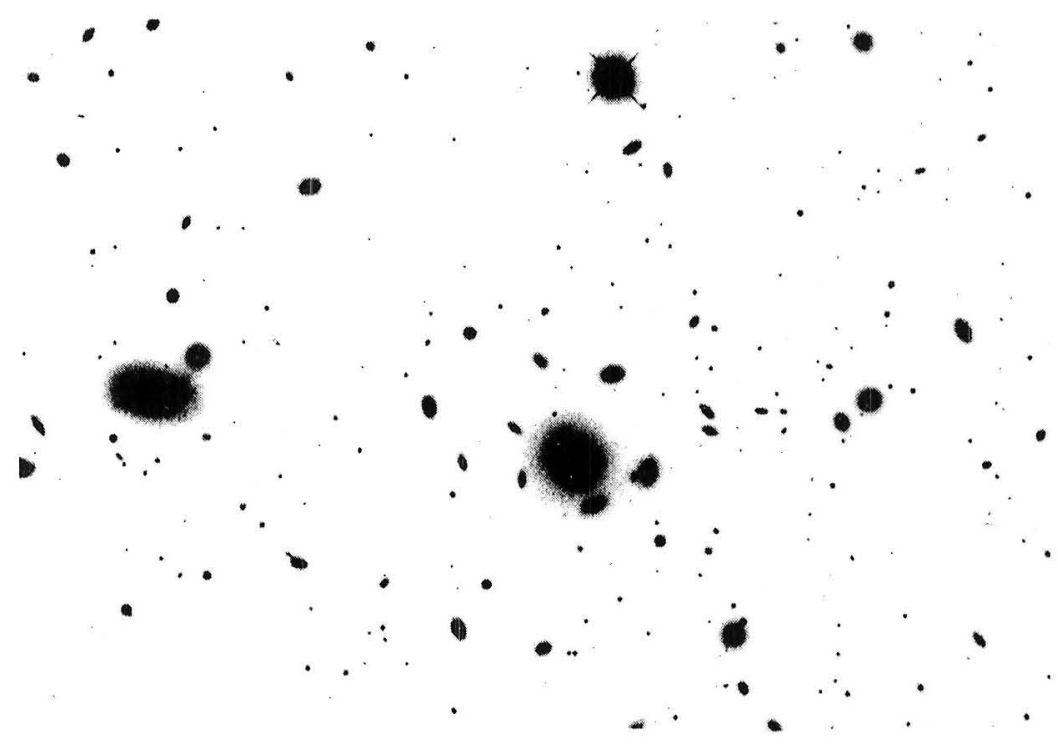

Figure 1

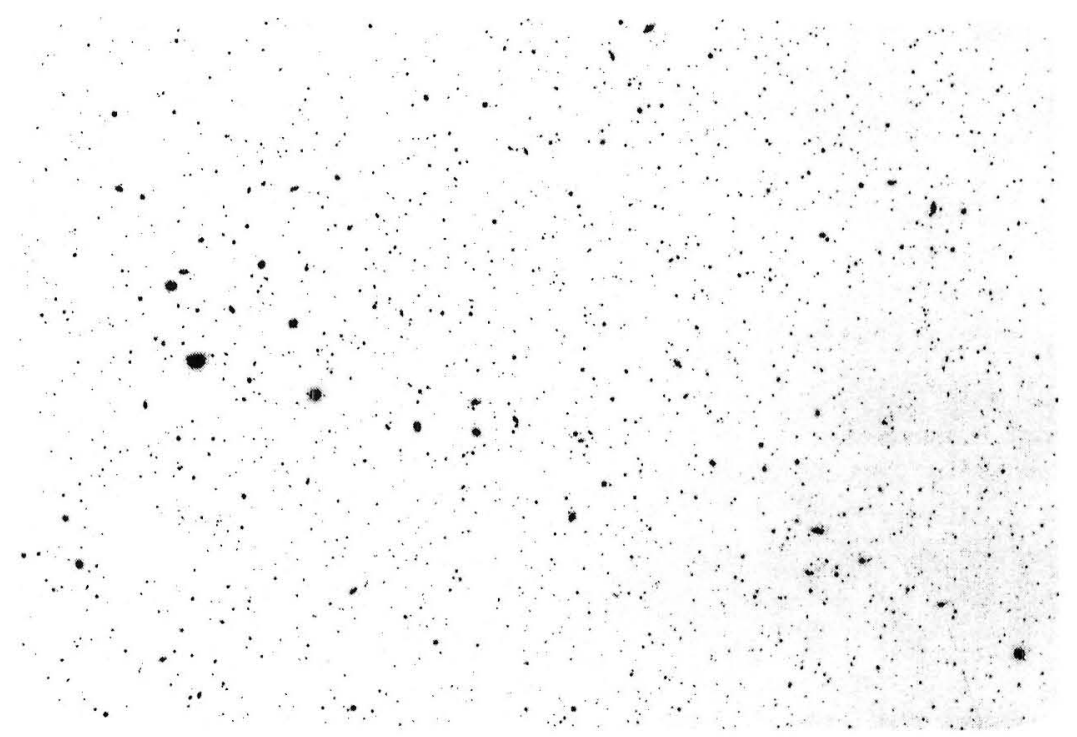

Figure 2 


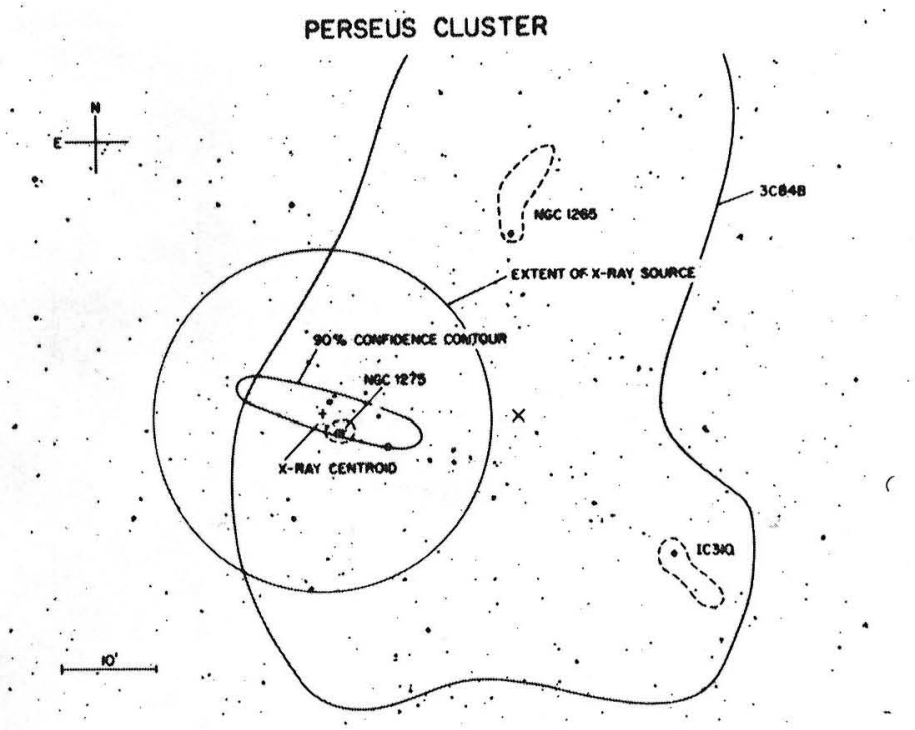

Figure 3
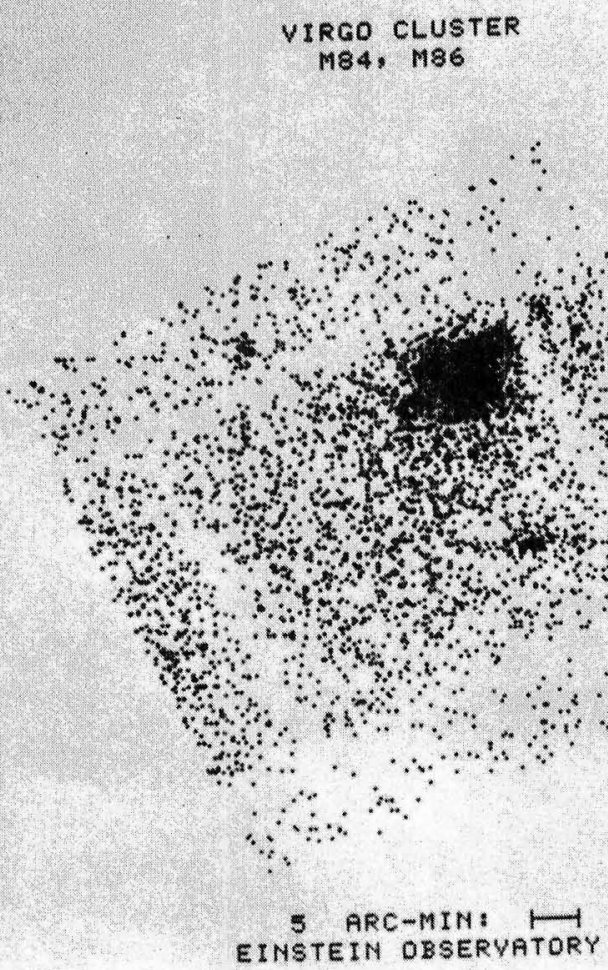

Figure 4 

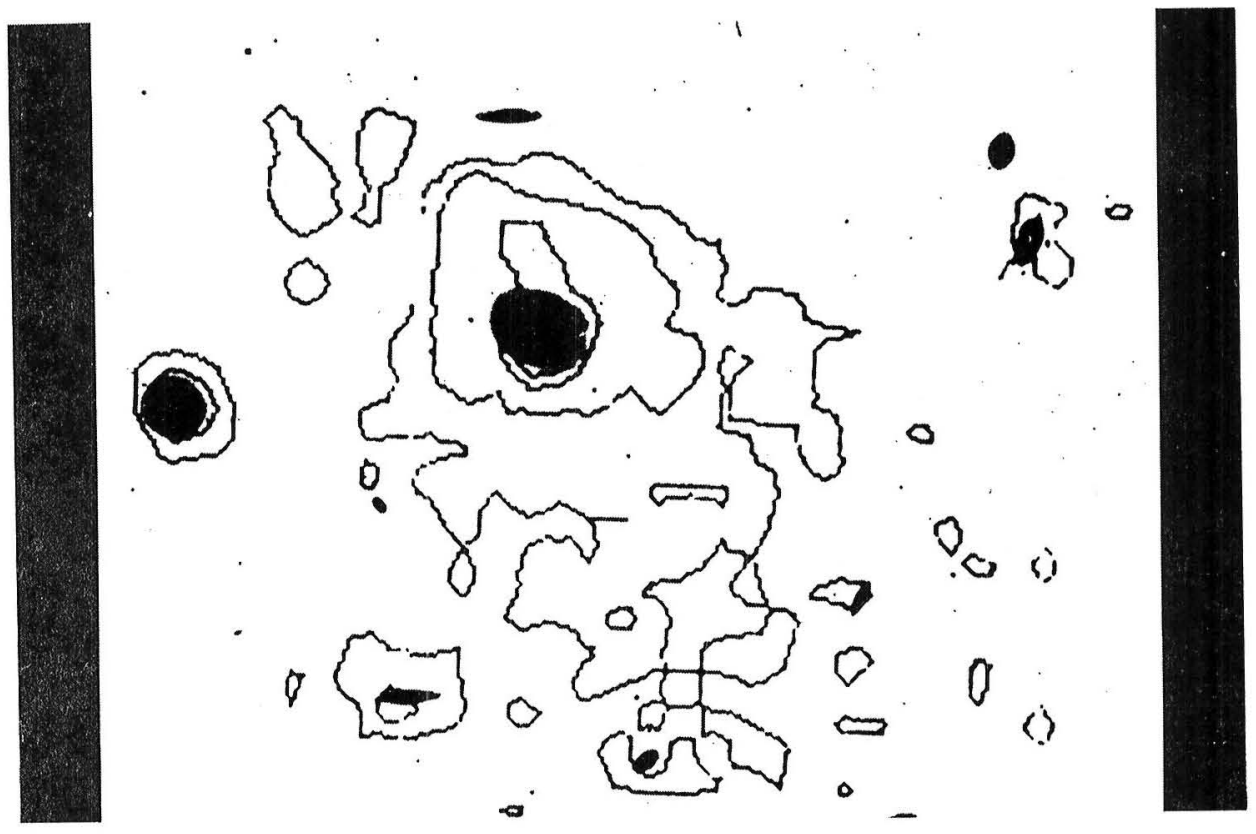

Figure 5

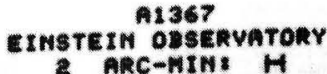

ARC-WIN: H
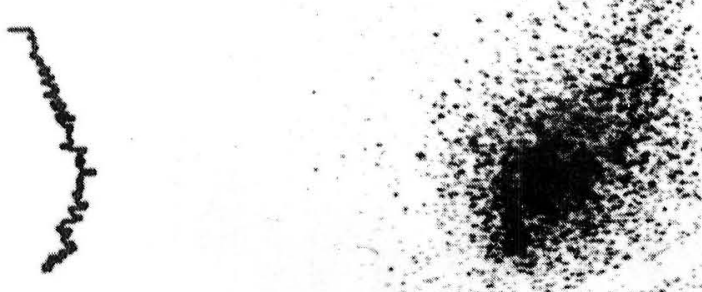

Figure 6 


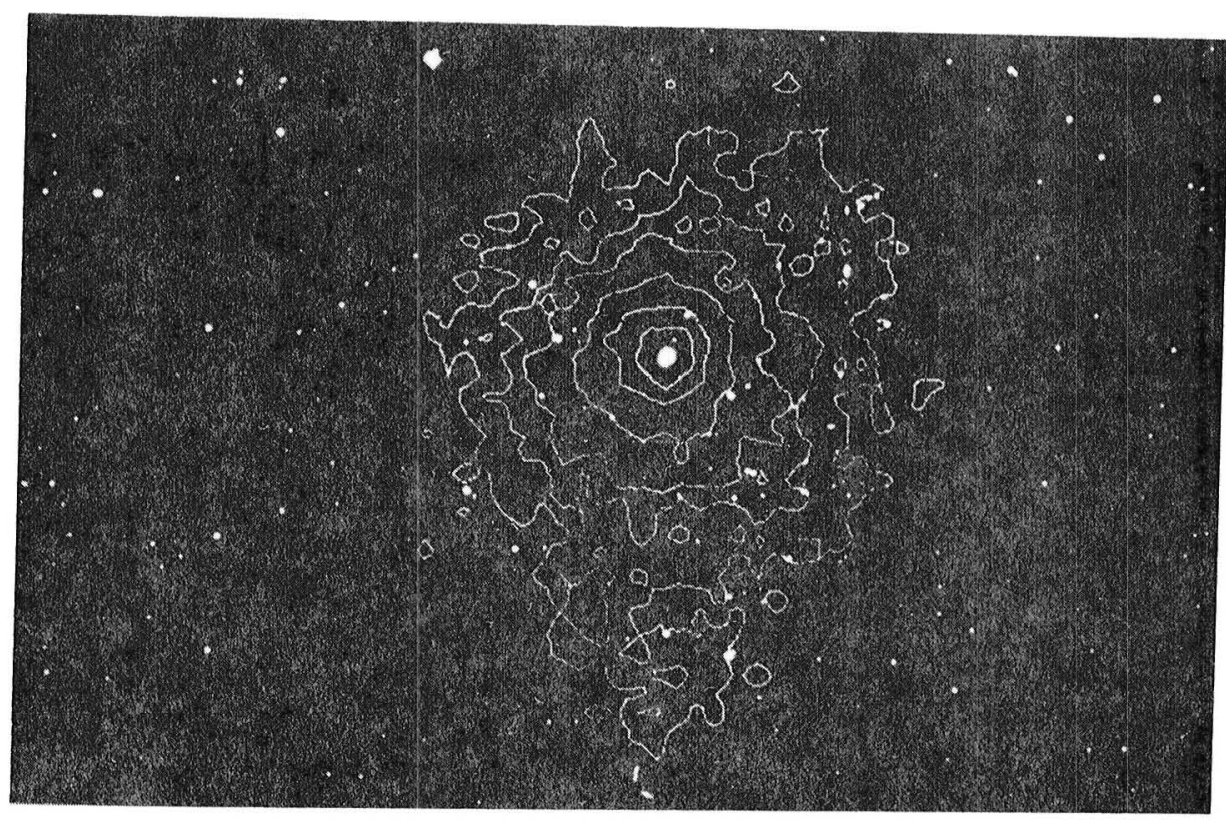

Figure 7
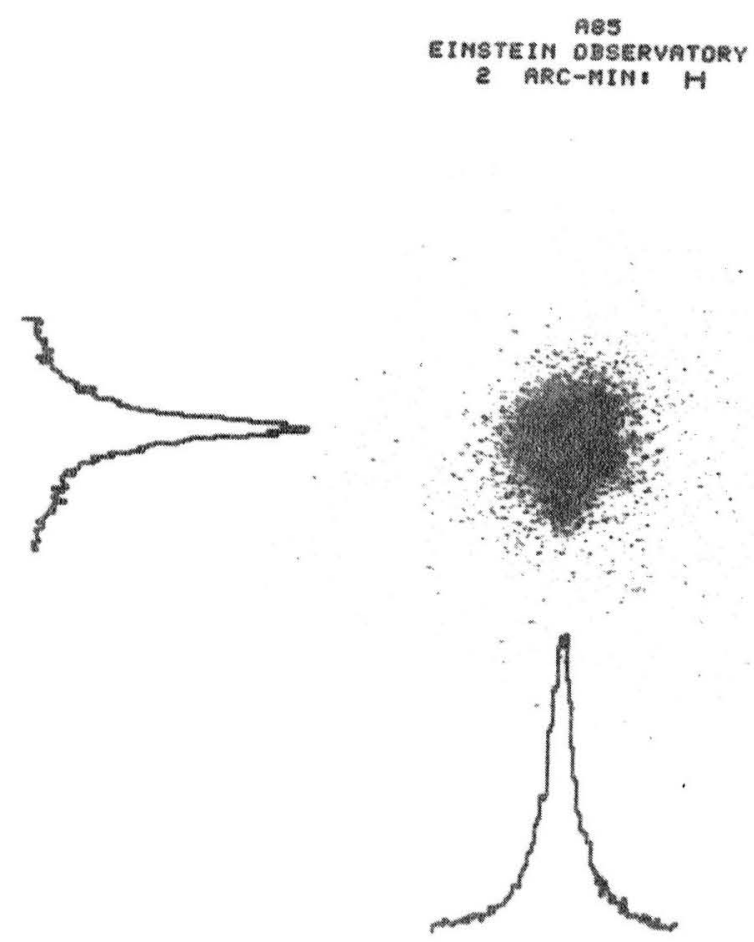

Figure 8 


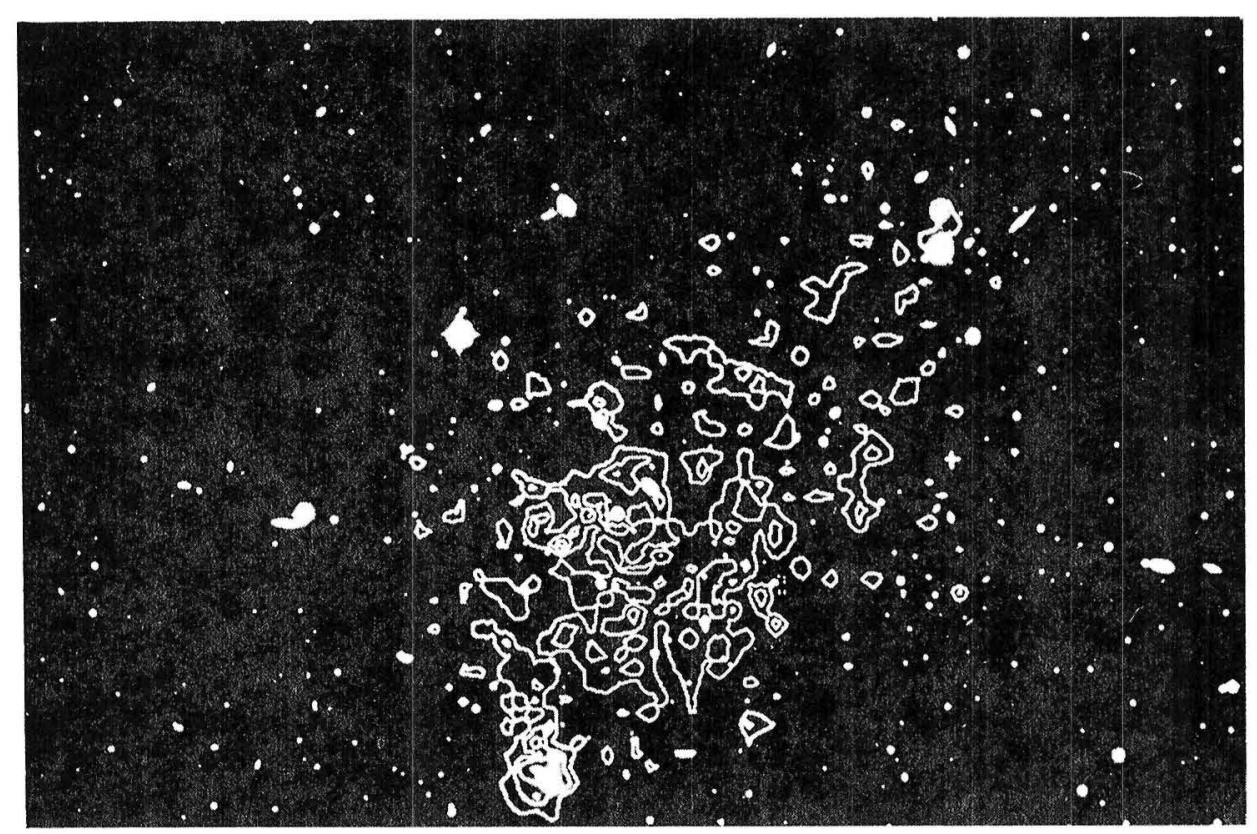

Figure 9 
品

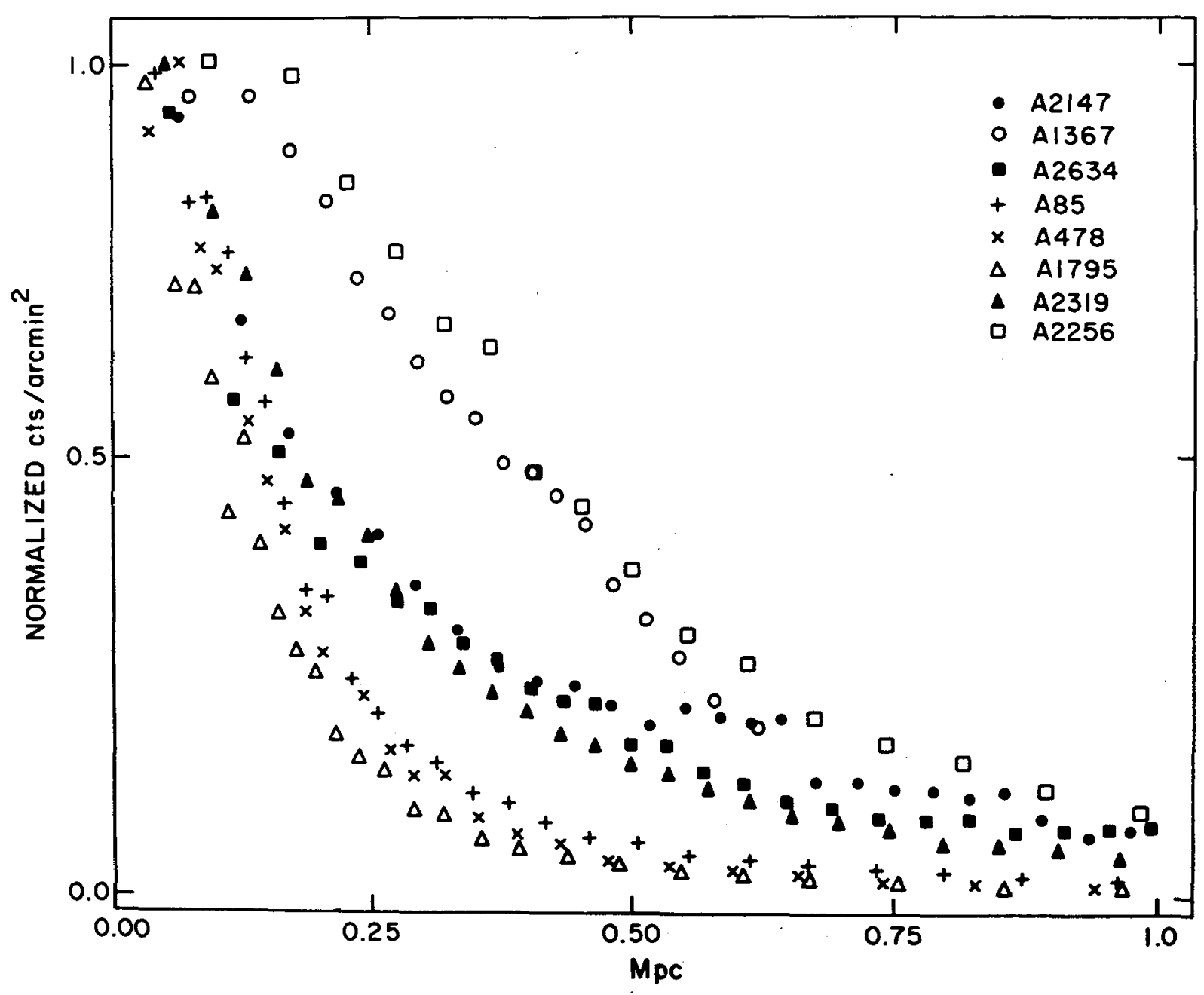

Figure 10 


\section{CLUSTER AROUND 3C295}

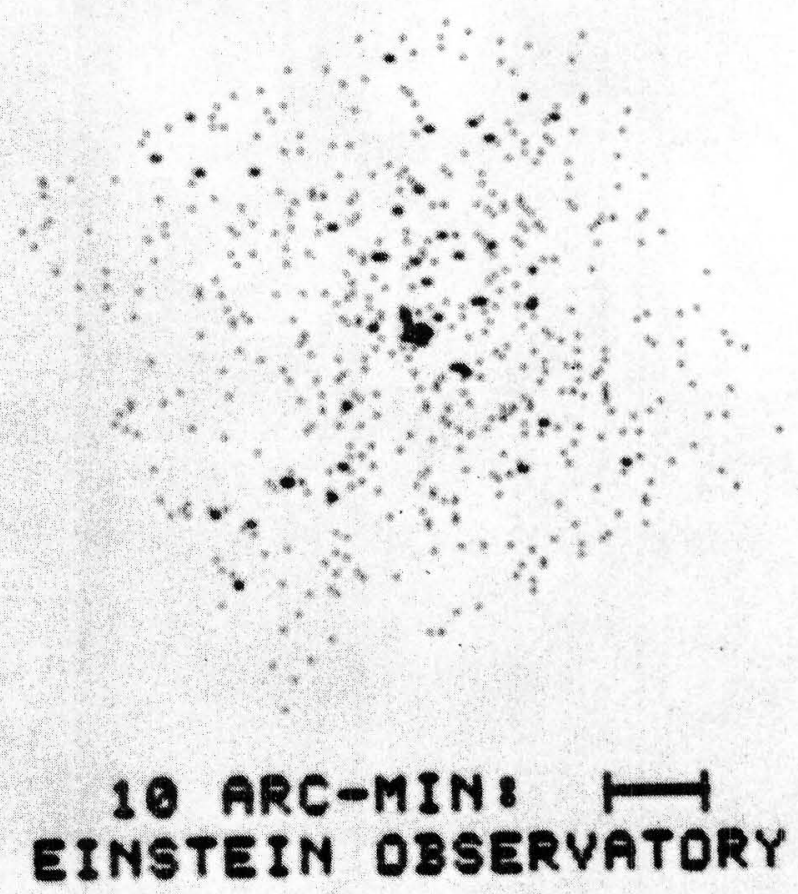

Figure 11 